\title{
High energy cosmic rays and thunderstorm connected radiations at the Tien Shan complex installation
}

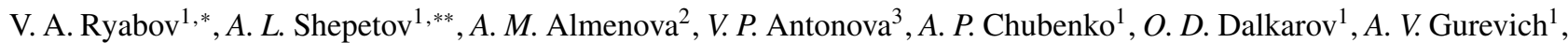

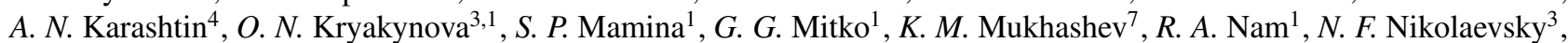

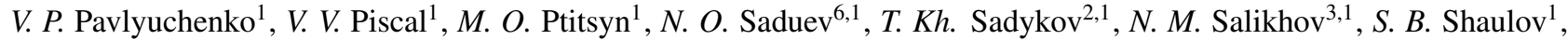 \\ $Y$ u. V. Shlyugaev ${ }^{5}, A$. V. Stepanov ${ }^{1}$, L. I. Vildanova ${ }^{1}$, M. I. Vildanova ${ }^{1}, N$. N. Zastrozhnova ${ }^{2}, Z h . S h . Z^{2}$ Zntayev $^{3}$, \\ V. V. Zhukov ${ }^{1}$, and $K . P$ Zybin $^{1}$ \\ ${ }^{1}$ P. N. Lebedev Physical Institute of the Russian Academy of Sciences (FIAN), 119991, Leninsky pr., 53, Moscow, Russia \\ ${ }^{2}$ Institute for Physics and Technology, Almaty, 050032, Ibragimova, 11, Kazakhstan \\ ${ }^{3}$ Institute of Ionosphere, 050020, Kamenskoye plato, Almaty, Kazakhstan \\ ${ }^{4}$ Research Radiophysics Institute, 603950, Bolshaya Pechyorskaya, 25/12a, Nizhny Novgorod, Russia \\ ${ }^{5}$ Institute of Applied Physics of RAS, 603950, Ul'yanova, 46, Nizhny Novgorod, Russia \\ ${ }^{6}$ Institute for Experimental and Theoretical Physics of al-Farabi Kazakh National University, al-Farabi pr., 71, Almaty, Kazakhstan \\ ${ }^{7}$ Abay Kazakh National Pedagogic University, Dostyk pr., 13, Almaty, Kazakhstan
}

\begin{abstract}
We present a general description as well as a sample of illustrative experimental results of the Tien Shan high altitude detector complex which is aimed for fundamental and applied investigations in different fields of astroparticle, atmospheric, and environmental physics. The unique natural conditions of the Tien Shan Mountain Scientific Station where this complex resides, the presence of a modern equipment, and the use of multi-messenger integrated approach by detection of various radiation types open a new stage of research in cosmic ray physics and high-energy atmospheric phenomena.
\end{abstract}

\section{Introduction}

The Tien Shan Mountain Cosmic Ray Station of the P. N. Lebedev Physical Institute is situated in the mountains of Northern Tien Shan (Kazakhstan Republic), at geographical co-ordinates $43^{\circ} \mathrm{N}, 75^{\circ} \mathrm{E}$, and at an altitude of $3340 \mathrm{~m}$ above the sea level. The program of scientific investigation which is currently realized here anticipates the activity in a wide range of problems connected with both physics of cosmic rays and with applied geophysics. Hence, the astroparticle group of experiments which is based on using the newly built extensive air shower (EAS) installation [1] involves the following directions of scientific research:

- precision measurements of the energy spectrum of the primary cosmic rays between $10^{14}$ and $10^{17} \mathrm{eV}$, the problem of the knee at $E_{0} \simeq 3 \cdot 10^{15} \mathrm{eV}$, and the search for local peculiarities in the primary spectrum;

- the lateral distribution of shower particles in the central EAS region;

- the small-scale anisotropy in the distribution of arrival directions of cosmic ray particles;

- the investigation of the hadronic EAS component as well as the connected low-energy neutron and gamma-

\footnotetext{
*e-mail: ryabov@x4u.lebedev.ru

**e-mail: ashep@tien-shan.org
}

ray shower accompaniment with a number of different energy thresholds;

- the investigation of the flows of the penetrative EAS component;

- the detection of EAS connected radio-emission in various wavelength ranges.

In analyzing EAS data special attention should be paid to the search for anomalous effects whose presence in the cosmic rays belonging to the energy range around the knee of the primary spectrum has been reported from time to time from various experiments held in former years both at Tien Shan station and at other sites.

In the range of geophysical research the following experimental tasks were considered for implementation at Tien Shan in the current decade:

- continuation of longstanding intensity variations registration of the low-energy cosmic rays $\left(E_{0} \gtrsim 10^{10} \mathrm{eV}\right)$ and implication of these data for prognosis of radiation conditions both in the near-Earth environment ("cosmic weather") and at the surface of the Earth;

- continuous monitoring of the intensity of the environmental background of soft gamma radiation and lowenergy neutrons as well as that of the high- and lowfrequency radio-emission, and the study of the possibility to use this information for the forecast problem of seismic activity events [2-4]; 
- the search for a possible interconnection between the cosmic rays and seismic effects within the Earth's crust which have been predicted earlier by some theoretical models [5, 6];

- detection and study of various radiation kinds which accompany the development of electric discharges in thunderclouds.

The subject of the present paper is an overview of some experimental facilities of the Tien Shan station with illustration of their capabilities by measurement data which have been obtained recently in studies held there in accordance with scientific research program mentioned above.

\section{Tien Shan shower installation and new results in the field of EAS physics}

The central part of the shower installation which is used at Tien Shan mountain station for EAS detection in the range of primary cosmic ray energies of $10^{14}-10^{17} \mathrm{eV}$ consists of a set of charged particles detectors whose sensitive part is a $0.5 \times 0.5 \times 0.05 \mathrm{~m}^{3}$ polystyrene scintillator block placed on the bottom of a pyramidal light-tight reflector, with a photomultiplier tube (PMT) residing at its top. Currently a set of 72 such detectors is installed in the nodes of rectangular grid with a uniform spatial step of $3 \times 4 \mathrm{~m}^{2}$, and with a total area of about $1000 \mathrm{~m}^{2}$ constituting a rather dense detector "carpet" which is aimed for precise investigations of the lateral distribution of shower particles flow within the EAS core region. The dynamic range of EAS particles density measurement by shower detectors is about $10^{5}$.

Synchronous operation of the whole detector system is provided by a control trigger signal which is elaborated in the passage moment of an EAS. The lowest EAS size level $N_{e}^{\min }$ of effective shower selection at the present time is set in such a way that showers with $N_{e} \gtrsim 3 \cdot 10^{5}$ (i.e. with primary energy $E_{0} \gtrsim 10^{15} \mathrm{eV}$ ) whose axes were hitting the central detector "carpet" can be registered with a nearly $100 \%$ probability, and detection of smaller showers up to $N_{e} \gtrsim(1-3) \cdot 10^{14} \mathrm{eV}$ is possible as well with a somewhat reduced efficiency.

Two-dimensional snapshots of particles flow registered in every EAS event by the central detector "carpet" permit to study later precisely the density distribution of the charged particles in extensive air shower in dependence on the distance from shower axis. A set of such lateral density distributions averaged over EAS events with close size values $N_{e}$ which have been detected during the recent, 2016-2017 years data collection runs is shown with separate circles in the figure 1. Analogue distributions which have been measured in a previous Tien Shan experiment Hadron (1985-1990, [7]) are plotted as continuous curves in this figure. In the range of shower core distances where both datasets do intersect with each other a rather good agreement is seen between the newly obtained and already known distributions. At the same time, recent measurements can be extrapolated much closer into the vicinity of the shower axis than was possible before which is a consequence of a much larger dynamic diapason of saturation-free scintillation amplitude measurement

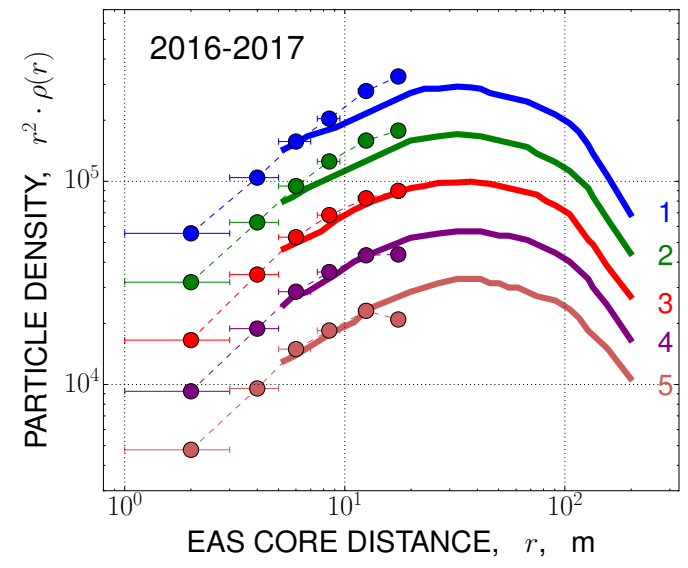

Figure 1. Lateral distribution of shower particles density in EAS with different sizes $N_{e}$ according to the present time measurements (circles) in comparison with analogous data of former Tien Shan experiments (continuous lines). Mutual correspondence between the numbers of plotted distributions and the range of EAS sizes is the following: $N_{e} \simeq 7.3 \cdot 10^{6}(1), N_{e} \simeq 4.1 \cdot 10^{6}$ (2), $N_{e} \simeq 2.3 \cdot 10^{6}(3), N_{e} \simeq 1.3 \cdot 10^{6}(4)$, and $N_{e} \simeq 7.3 \cdot 10^{5}(5)$.

at modern ADC system in comparison with what has been accessible earlier.

Amongst various kinds of additional detector facilities which operate together with the shower installation at Tien Shan there is a set of neutron detectors for the measurement of the low-energy neutron flux after the passage of extensive air showers. The detectors are based on the $\varnothing 30 \times 1000 \mathrm{~mm}^{2}$ gas discharge counters grouped by 6 pieces in a number of separate detector modules. Some of these counters are surrounded by cylindrical tubes of neutron moderator subsistence (paraffin, polyethylene) which permits to enlarge the energy range of detected neutrons from thermal energies $\left(E_{n} \sim 10^{-2} \mathrm{eV}\right)$ up to $E_{n} \sim 10^{3}-10^{5} \mathrm{eV}$. The efficiency of neutron detection for different moderator types was defined through Geant4 simulation of neutron interactions within the detector material for a number of corresponding detector models.

The average multiplicity distributions of neutron counts detected in EAS events dependent on the range of shower size value $N_{e}$, and on distance $R$ between the location of neutron detector and the EAS axis are presented in the figure 2. Lateral distributions in the plots of this figure are limited from above with a maximum distance value $\simeq 36 \mathrm{~m}$ which is defined by the geometrical dimensions of central detector "carpet" of the shower installation. It is seen that generally the shape of the lateral distribution functions of neutron signal remains one and the same in the events with $N_{e} \lesssim 10^{6}$, but their slope starts to diminish rapidly fors larger EAS whose size exceeds this limit.

Taking into account the typical probability of neutron registration $\varepsilon \approx 15-20 \%$ which was defined by Geant 4 simulation of neutron interaction processes in the considered detector, and the effective sensitive area of the latter $S$ one can calculate the mean absolute flux of neutrons which have passed through the detector after an EAS hit. These estimations are shown along the right axes of the plot in 


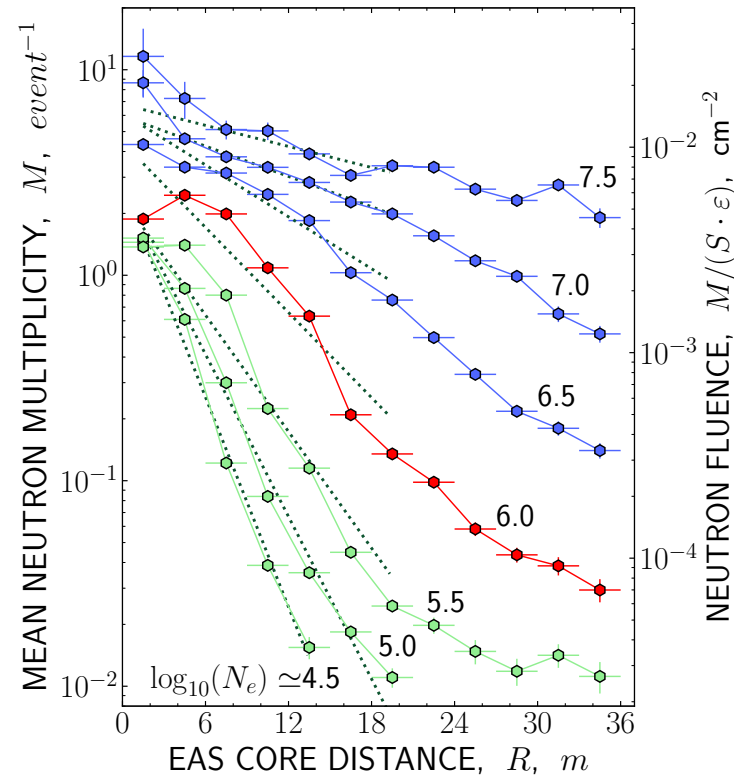

Figure 2. Lateral distributions of the mean multiplicity and corresponding integral flux of low-energy (thermal) neutrons around the EAS core region. The numbers above the curves is the average decimal logarithm of the size of the corresponding showers $N_{e}$.

figure 2. As seen there, the integral flux of low-energy neutrons in the core region of $N_{e} \simeq 10^{4}-10^{7}$ EAS varies within the limits of $10^{-4}-10^{-2} \mathrm{~cm}^{-2}$, in dependence on the size of the shower and the distance between its center and the point of detector location.

\section{Radiation measurements in thunderstorm time}

A longstanding experimental task which has been fulfilled during last decade at the Tien Shan mountain station is the investigation of various kinds of radiation emitted by the development of electric discharges in the Earth's atmosphere [8]. Currently, a special observation point has been created for this purpose on the top of a neighbouring mountain ridge in the vicinity of the station, at an altitude of $\sim 400 \mathrm{~m}$ above its common level (at an absolute height of $3750 \mathrm{~m}$ a.s.1.). At the time of thundercloud passage this point often occurs to be immersed immediately within the clouds which increases the probability for accelerated charged particles and accompanying gamma radiation to reach the detector system. The basic detector types applied in this point is a set of gamma ray detectors on an inorganic $\mathrm{NaI}(\mathrm{Tl})$ scintillator, and a full absorption spectrometer for detection of the charged particles by coincidence signal from a number of big $\left(1 \times 1 \mathrm{~m}^{2}\right)$ plastic scintillator plates interlaced with sheets of absorber material. Together with these particle detectors a number of radio-, electric field, and acoustic sensors are used to trace particularly the development of atmospheric electric discharges at different time scales.

Figure 3 illustrates the response of the high altitude particles detector system on various electric phenomena in the atmosphere. In the left panel of this figure it is shown a set of signal intensity records from different detectors taken shortly after a close lightning discharge which has then occurred at a distance of about 30-100 m from this detector. Among these records in the ELECTRONS plot an imprint of a momentary charged particles increase is clearly seen accompanied by a simultaneous outburst of hard gamma radiation in a number of GAMMA time series taken with different energy thresholds. The moment of electron/gamma burst is delayed up to $\sim 100$ milliseconds relative to the beginning of the discharge development which can be traced by the time history of its 5$30 \mathrm{MHz}$ radio emission, and by the fast variation of the local electric field, seen correspondingly in the RADIO and ELECTRIC FIELD series. Another type of electric discharge connected events is presented in the middle panel of figure 3 where a prolonged increase of gamma radiation intensity is seen whose duration is much larger, and its energy spectrum is much softer than in the previous case. Lastly, in the rightmost panel of figure 3 is shown the time behaviour of local gamma radiation intensity registered during a thunderstorm ground enhancement (TGE) event which was caused by the close thundercloud passage immediately above the detector point [9].

An alternative informational channel on the processes which accompany development of atmospheric electric discharges is connected with the optic radiation of visible lightning flashes. A special pair of optic sensors was applied in the last years at the Tien Shan station to detect the lightning emission in the near ultraviolet and the red/near infrared wavelength ranges of electromagnetic spectrum [10].

The most common behaviour of the electromagnetic radiation flash in the time of lightning process can be seen in the top panel of figure 4 . The starting moment of the atmospheric discharge here can be defined by the signal of its radio emission in the $R A D I O$ plot, but up to times of 250-300 ms after initiation the discharge remains "dark", i.e. any detectable signal is absent in both INFRARED and ULTRAVIOLET time series. After this delay the optic emission starts to be observable, its thin structure consisting mostly of narrow intensive outbursts which generally coincide with similar emission bursts in the radiofrequency wave range. Besides such transient bursts there is a period of continuous optical emission near the maximum of the discharge development which is accompanied with a detectable fast variation of the local electric field. The whole time history of the discharge has a quite perceptible duration and can last about 500-800 ms. Another type of lightning phenomena is connected with very short emission bursts which last only a few milliseconds only, like the one presented in the bottom panel of figure 4 .

\section{Acknowledgments}

This work is supported by the grants \#BR05236201 and \#BR05236494 of IRN Program "Fundamental and applied studies in related fields of the physics of terrestrial, nearEarth and atmospheric processes and their practical application", and by the grant \#0118RK00800 of the Cosmic Program of Kazakhstan Republic. 

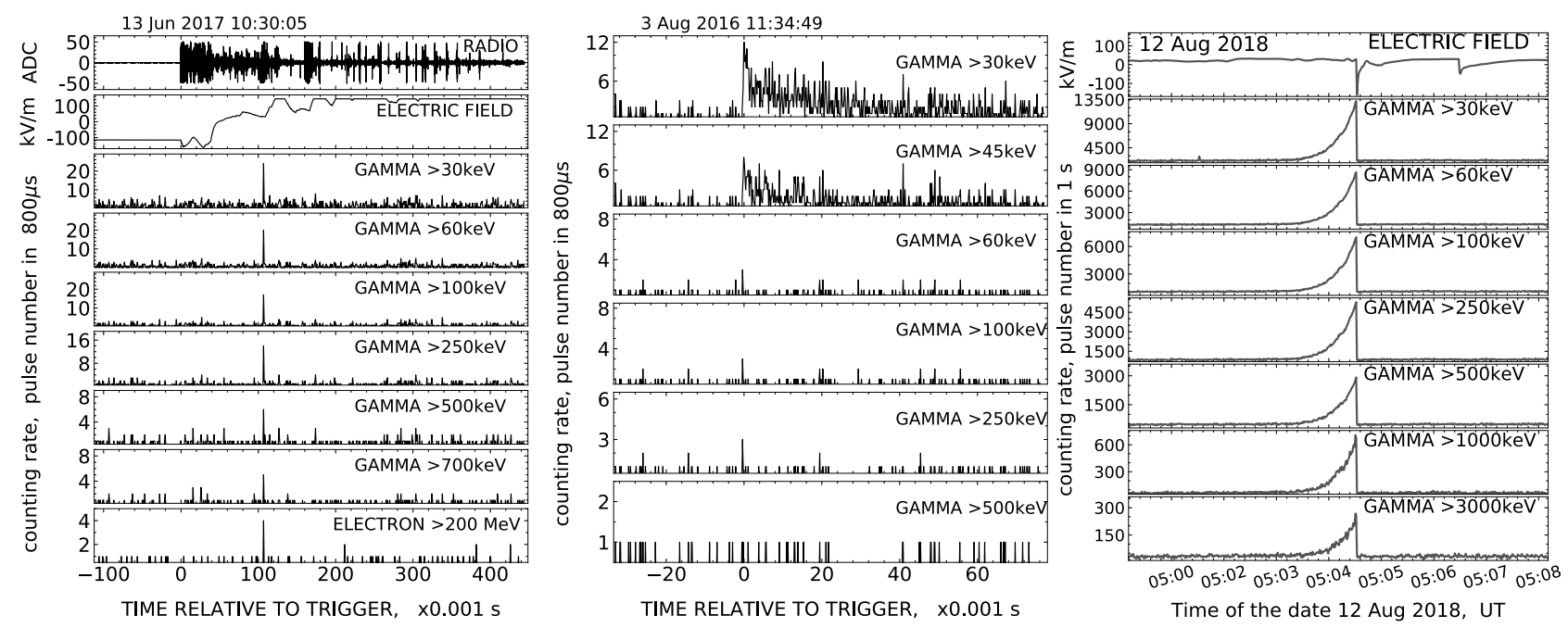

Figure 3. Example of physical results gained at the high altitude detector point. Left: typical imprint of a short electron/gamma avalanche which has occurred at lightning moment. Middle: a transient prolonged flash of the soft gamma-radiation observed in thunderstorm time. Zero point of time axes both in the left and middle panels corresponds to beginning of discharge development. Right: a TGE event observed in time of a close thunderstorm.

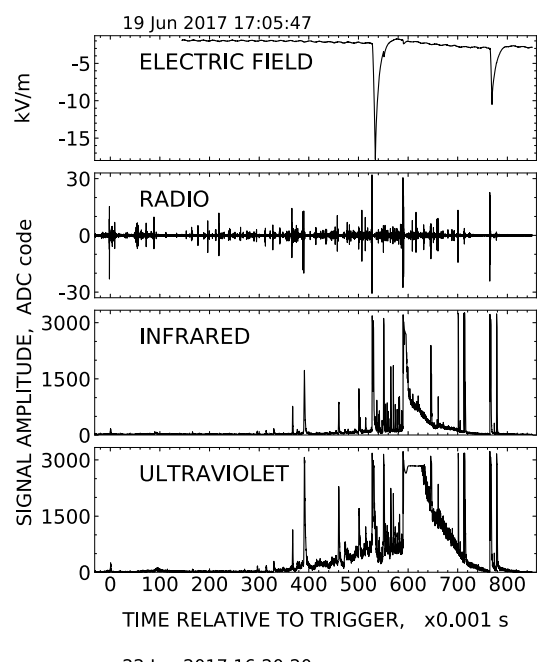

\section{References}

[1] A.P. Chubenko, A.L. Shepetov, V.P. Antonova et al., Nucl. Instrum. Methods A 832, 158 (2016)

[2] V.P. Antonova, L.I. Vildanova, A.V. Gurevich et al., Geomagnetizm and Aeronomy 49, 761 (2009)

[3] N. Salikhov, G. Pak, O. Kryakunova et al., An increase of the soft gamma-radiation background by precipitations, in Proceedings of the 32nd ICRC (Beijing, China, 2011), Vol. 11, pp. 368-371

[4] N. Salikhov, A. Shepetov, A. Chubenko et al., Observation of the possible prior earthquake effect on the intensity of low-energy neutrons, gamma-radiation, and on the local electric field in Tien Shan mountain (2013), arXiv: 1301.6965 [physics.geo]

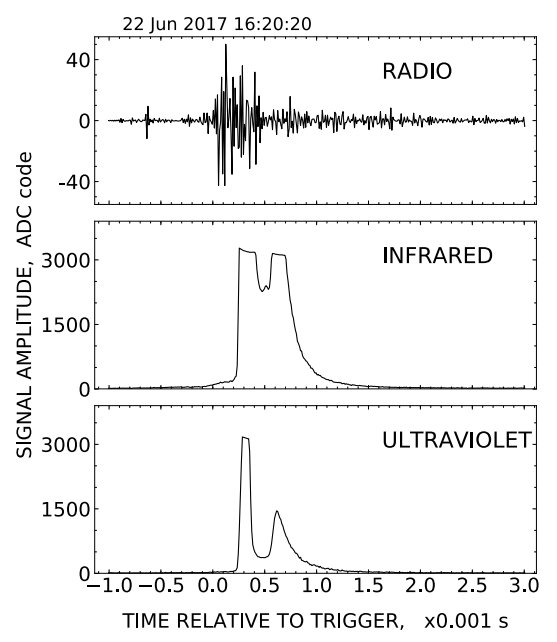

[5] O.B. Chavroshkin, A.V. Nikolaev, L.N. Rykunov, V.V. Tsyplakov, Methods, results and perspectives of the high frequency seismic noise and vibrosignals, in 6th Rep IASPEL Comm. Microseismol. 18 IUGG (Hamburg, Germany, 1983)

[6] V.A. Tsarev, V.A. Chechin, Atmospheric muons and high-frequency seismic noise, LPI Preprint No. 179 (1988)

[7] S.F. Abdrashitov, D.S. Adamov, V.V. Arabkin et al., Bull. Acad. Sci. USSR Phys. Ser. 50, 123 (1986)

[8] A. Gurevich, A. Almenova, V. Antonova et al., Phys. Rev. D 94, 023003 (2016)

[9] A. Chilingarian, S. Chilingarian, T. Karapetyan, et al., Sci Rep 7, 1371 (2017)

[10] A. Gurevich, G. Garipov, A. Almenova et al., Atmos. Res. 211, 73 (2018)

Figure 4. High resolution records of the lightning discharge connected electromagnetic radiation in radio and optic wave ranges. Zero point of the time axes corresponds to beginning moment of electric discharges. 